\title{
Modeling the Impact of Vaccination, Screening, Treatment on the Dynamics of Pneumonia
}

\author{
K. S. Rahman ${ }^{1 *}$, S. R. Mitkari ${ }^{1}$, S. Shaikh ${ }^{2}$ \\ ${ }^{1}$ Department of Physics, Shri Siddheshwar College, Majalgaon, Beed (M.S.), India \\ ${ }^{2}$ Department of Mathematics, Maulana Azad College, Aurangabad - 431001 (M.S.), India
}

Received 9 March 2020, accepted in final revised form 9 June 2020

\begin{abstract}
In this paper we have presented a deterministic model for pneumonia transmission and we have used the model to avail the potential impact of therapy. The model is based on the vaccinated-susceptible-carrier-infected-recovered-susceptible compartmental structure and their possible interventions with the possibility of infected individual recovery from natural immunity. Here, we have modeled Pneumonia considering vaccination, screening and treatment with a system of nonlinear ordinary differential equation. The model reproduction number $\mathrm{R}_{0}$ is derived and the stability of the equilibria are derived. The stability of equilibrium points is analyzed. The results shows that there exists a locally stable disease free equilibrium points, $E_{0}$ when $R_{0}<1$ and a unique endemic equilibrium $E_{1}$, when $R_{0}>1$. Infection free point was found to be locally stable and if reproduction number is greater than unity, then there is unique endemic equilibrium point and if it is less than unity, the endemic equilibrium point is globally asymptotically stable and pneumonia will be eliminated.
\end{abstract}

Keywords: Mathematical modelling; Pneumonia; Reproduction number; Endemic equilibrium.

(C) 2020 JSR Publications. ISSN: 2070-0237 (Print); 2070-0245 (Online). All rights reserved. doi: http://dx.doi.org/10.3329/jsr.v12i4.45815

J. Sci. Res. 12 (4), 525-536 (2020)

\section{Introduction}

Pneumonia is an infection of the lungs that is caused by bacteria, viruses, fungi, or parasites which is characterized primarily by inflammation in the lungs or by alveoli that are filled with fluid. Bacteria and viruses are the primary causes of pneumonia. When a person breath pneumonia causing pathogens into his lungs, and the body's immune system cannot prevent entry, the organisms settle in the small air sacs called alveoli and continue to multiply. The host body sends white blood cells to attack the infection causing the sacs to be filled with fluid and pus causing pneumonia. The people most susceptible to pneumonia are the old, infant, the sick and those with impaired immune systems [1].

*Corresponding Author: sanarahman454@gmail.com 
Protozoal and helmenthic parasitic pnuemonia and lung involvement are common in tropics with few exceptions, they most commonly occur in the western world and are diseases of immune-compromised hosts [2]. At the present time world is facing the crisis of novel infectious disease called COVID-19.Most people who get COVID-19 have mild or moderate symptoms like coughing, a fever, and shortness of breath. But some who catch the new coronavirus get severe pneumonia in both the lungs. COVID-19 pneumonia is a serious illness that can be deadly [3].

Pneumococus spreads through contact with people who are ill or who carry the bacteria in their throat. It is common for people, especially children, to carry bacteria in their throat without being sick. After a person is infected and diagnosed with pneumonia, he will be on medication for a particular period of time, the infection is contagious for 10 to 14 days after the infected person stops getting treatment [4].

Prevention of pneumonia includes vaccination, environmental measures, screening and appropriately treating the diseases. Suctioning the mouth and throat of infant with micronium stained amniotic acid decreases the rate of aspiration pneumonia. Environmental prevention methods include reduction indoor air pollution as well as smoke cessation and hand-washing when around a person with pneumonia, since the bacteria and viruses can also spread through ones hands and then to the mouth [5]. The present vaccination for pneumonia fails in the case of coronavirus pneumonia. Parasitic pneumonia is mostly treated with anti-parasitic or sometimes deworming methods. Many anti-parasitic drugs are on trial for the probable treatment of COVID-19 as one such is ivermectin [6]. Some of the precautionary measure as school deworming programs which was initiated by WHO, that could be an alternative to curb these diseases. Probiotics is one more option for precautionary measure as probiotics remain a promising method to prevent ventilator associated pneumonia (VAP) and other infections in critically ill patients, with biological plausibility clinical promise and apparent cost effectiveness based on data available to date [7].

Huang et al. [8], Lipsitch et al. [9] and Otieno et al. [10], have done research on the dynamics of pneumonia. In this paper, we have considered the weak nature of immune system which is vulnerable to different types of pneumonia as long as they coexist in population. It also considers the effects of treatment and the likelihood of wrong treatment due to similar symptom. It stresses the need of vaccinating, proper screening, proper treatment to avoid relapse and boosting of natural immunity to eradicate pneumonia. Here, we have formulated a model based on vaccination screening and treatment and different probabilities. And here we will try to find possible ways to curb pneumonia and to some extent coronavirus which is a major problem the world is facing now.

This paper is structured as follows. In section 2, the model is formulated based on the assumptions and definitions of variables and parameters. In section 3, model analysis where we have analyzed the model based on reproduction number and stability analysis of the model. In section 4, sensitivity analysis of the model is available to analyze the sensitive parameters of the model for the transmission dynamics of pneumonia. Finally in section 4 , we discuss the results and make a conclusion. 


\section{Model Formulation and Description}

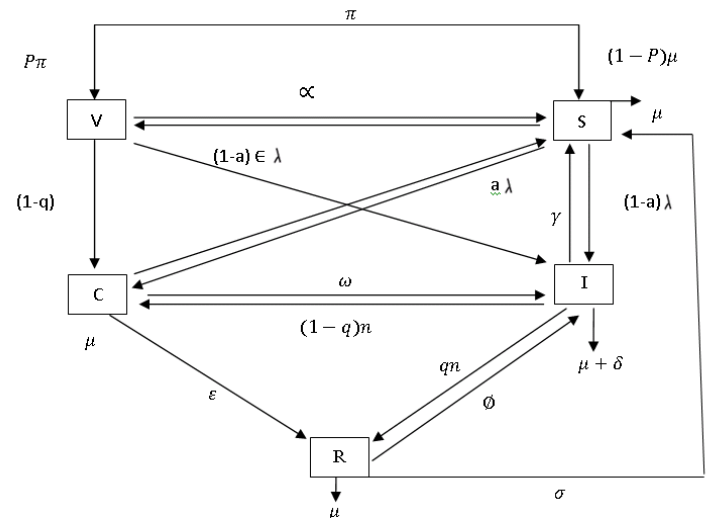

Fig. 1. A Compartmental Diagram for the Pneumonia Transmission dynamics.

\section{Model Equations}

$$
\begin{aligned}
& \frac{d v}{d t}=p \pi+\beta s-(\alpha+\epsilon \lambda+\mu) V \\
& \frac{d s}{d t}=(1-p) \pi+\alpha V+\gamma C+v I+\sigma R-(\beta++\mu) s \\
& \frac{d t}{d t}=\in a \quad V+a \quad s+(1-q) n I-(\gamma+\omega+\varepsilon+\mu) c \\
& \frac{d t}{d t}=(1-a) \quad S+(1-a) \in \lambda V+w c+\emptyset R-(v+n+\mu+\delta) I \\
& \frac{d t}{d t}=\varepsilon c+q n I-(\varnothing+\sigma+\mu) R \\
& \text { With initial condition } \mathrm{S}(0)=\mathrm{S}_{0},\left(\mathrm{~V}_{0}\right)=\mathrm{V}_{0}, \mathrm{C}(0)=\mathrm{C}_{0,} \mathrm{I}(0)=\mathrm{I}_{0}, \mathrm{R}(0)=\mathrm{R}_{0}
\end{aligned}
$$

Table 1. Description of Variable and parameters of the model.

\begin{tabular}{ll}
\hline Variable & Description \\
\hline $\mathrm{V}(\mathrm{t})$ & No. of vaccinated individuals at time $\mathrm{t}$ \\
$\mathrm{S}(\mathrm{t})$ & No. of susceptible individuals at time $\mathrm{t}$ \\
$\mathrm{C}(\mathrm{t})$ & No. of Carrier individuals at time $\mathrm{t}$ \\
$\mathrm{I}(\mathrm{t})$ & No. of infected individuals at time $\mathrm{t}$ \\
$\mathrm{R}(\mathrm{t})$ & No. of recovered individuals at time $\mathrm{t}$ \\
\hline
\end{tabular}

\begin{tabular}{cl}
\hline Parameters & Interpretation \\
\hline$\pi$ & Recruitment rate \\
$\mu$ & Natural death rate \\
$\delta$ & Disease induced death rate for I class \\
$\lambda$ & Force of infection \\
a & Probability that newly infected individuals are asymptomatic / carrier. \\
$\alpha$ & Waning rate of vaccine \\
$\beta$ & Rate of vaccination from $\mathrm{S}$ to $\mathrm{V}$ \\
$\in \lambda$ & Rate of vaccinated getting carrier and infected \\
$\gamma$ & Rate at which carrier transform to susceptible class \\
$\omega$ & Rate at which carrier transform in infected class \\
$\eta$ & Rate of infected getting carrier and infected. \\
$\xi$ & Recovery rate due to prompt treatment
\end{tabular}


$\varnothing \quad$ Recovery rate due to infected class

$\mathrm{v} \quad$ Rate at which infected transferring to susceptible class.

$\sigma \quad$ Rate at which recovered person getting susceptible.

The model divides the total population into five subclasses namely susceptible $S(t)$, Vaccinated $V(t)$, Carrier $C(t)$, Infected $I(t)$ and recovered $R(t)$. The individuals are recruited into the vaccinated and susceptible class either by immigration or by birth rate $\pi$. Let $\mu$ be the natural death at any compartment. Let $p \pi$ be the number of vaccinated persons. Let $(1-p) \pi$ be the susceptible number of people. Since vaccines wanes with time the protected individuals after its expiry return backs to susceptible compartment at the rate $\alpha$. Individuals move from susceptible class to vaccinated class with vaccination rate of $\beta$. The susceptible class is infected either by carrier or symptomatically infected individuals with a force of infection

$\lambda=x\left(\frac{I(t)+p c(t)}{N}\right)$

where $x=K \tau, K$ is contact rate, $\tau$ is the probability that contact is effective to cause infection and $\rho$ is transmission coefficient for the carrier. If $\rho>1$ then, the carriers infect susceptible more highly than infective. If $\rho=1$, then both carriers and infective have good chance to infect susceptible than carriers. It is assumed in the model that vaccinated class $(V)$ also has a chance of transferring to infection or carrier classes with small proportion. The force of infection for the vaccinated class be $\lambda_{u}=\lambda \epsilon$ where $0 \leq \epsilon<1$ and $\epsilon$ is the proportion of the serotype not covered by the vaccine. Newly affected individuals by the force of infection become either carrier with a probability of ' $a$ ' and joins the carrier class $C$ or move to the infected class I with probably of ' $1-a$ '. The carrier class can develop and join the infected class with a rate of $\omega$ or recover by gaining natural immunity at $\varepsilon$ rate. Individuals in the infected class move to recovered compartment at a per capita rate of $n$ by treatment, with treatment efficiency of $q$ proportion of individuals join the recovered class or join the carrier class with $(1-q)$ proportion by adapting the treatment, or die from the disease at the rate $\delta$. Individuals from recovered class lose their temporary immunity by $\sigma$ rate. Recovery rate from infected class be $\varnothing$. Let $\gamma$ be the rate at which carriers gets back to susceptible class.

\section{Model Analysis}

\subsection{Equilibrium states of the model/the disease free equilibrium (EFE)}

The disease free equilibrium of model (1) to (5) is obtained by equating it to zero.

i.e. $\frac{d V}{d t}=\frac{d S}{d t}=\frac{d C}{d t}=\frac{d I}{d t}=\frac{d R}{d t}$

and in the absence of disease.

$\mathrm{I}=0, \mathrm{C}=0, \mathrm{R}=0$ reads to

$P \pi+\beta S_{0}-(\alpha+\mu) V_{0}=0$ 
$(1-P) \pi+\alpha V_{0}(\beta+\mu) S_{0}=0$

then by rearranging (6) and (7) and after substituting each other, we get

$S_{0}=\frac{(\mu-P \pi \mu+\alpha) \pi}{\mu(\mu+\alpha+\beta)}=\frac{\pi}{\mu} A$,

Where $\mathrm{A}=\frac{(\mu-P \pi \mu+\alpha)}{(\mu+\alpha+\beta)}$

$V_{0}=\frac{(P \pi \mu+\beta) \pi}{\mu(\mu+\alpha+\beta)}=\frac{\pi}{\mu} B$

where $\mathrm{B}=\frac{P \pi \mu+\beta}{\mu+\alpha+\beta}$

Then the disease free equilibrium is given by,

$E_{0}=\left(\frac{\pi}{\mu} B, \frac{\pi}{\mu} A, 0,0,0\right)$

\subsection{The effective reproductive number (Reff)}

The threshold parameter that governs the spread of a disease is called the effective reproduction number. The reproduction number is calculated by next generation matrix method as follows [11,12]:

The model equations are:

$\frac{d C(t)}{d t}=\epsilon a \lambda V+a S+(1-q) n I-(\gamma+\omega+\epsilon+\mu) n I-(\gamma+w+\varepsilon+\mu) C$
$\frac{d I(t)}{d t}=(1-a) S+(1-a) \epsilon \lambda V+\omega c+\emptyset R-(v+n+\mu+\delta) I$

by the principle of next generation matrix, we get,

$\left[\begin{array}{c}\epsilon a \lambda V+a \lambda S \\ (1-a) S+(1-a) \epsilon\end{array}\right]$
$\left[\begin{array}{c}(v+\omega+\varepsilon+\mu)(-(1-q) n I \\ (v+n+\mu+\delta) I-\omega c\end{array}\right]$

Since $\lambda=\mathrm{bC}+\mathrm{dI}$

Where $b=K \tau P$ is transmission co-efficient for the carrier compartment and $d=k \tau$ is also the transmission co-efficient for the infective compartment. The Jacobian of matrix $f$ and $v$ with respect to $c$ and $I$ at the disease free equilibrium $\left(V_{0}, S_{0}, 0,0,0\right)$ is as follows:

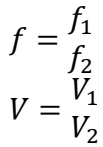

where $\mathrm{f}_{1}=\epsilon a \lambda V+a \lambda S$

$\mathrm{f}_{2}=(1-a) \lambda S+(1-a) \epsilon \lambda V$

$\mathrm{f}_{3}=(v+\omega+\varepsilon+\mu)(-(1-q) n I$

$\mathrm{f}_{4}=(v+n+\mu+\delta) I-\omega c$

$F=\left[\begin{array}{ll}\frac{d f_{1}}{d c} & \frac{d f_{1}}{d I} \\ \frac{d f_{2}}{d C} & \frac{d f_{2}}{d I}\end{array}\right] ; \quad V=\left[\begin{array}{ll}\frac{d V_{1}}{d c} & \frac{d V_{1}}{d I} \\ \frac{d V_{2}}{d C} & \frac{d V_{2}}{d I}\end{array}\right] ;$

$\frac{d f_{1}}{d c}=\epsilon a b V+a b s$

$\frac{d f_{1}}{d I}=\epsilon a d V+a d s$ 
$\frac{d f_{2}}{d c}=(1-a) b V+(1-a) \epsilon b V$
$\frac{d f_{2}}{d I}=(1-a) d s+(1-a) \epsilon d V$

Similarly,

$\frac{d V_{1}}{d c}=\gamma+w+\varepsilon+\mu$

$\frac{d V_{1}}{d I}=-(1-q) n$

$\frac{d V_{2}}{d C}=-\omega$

$\frac{d V_{2}}{d I}=\gamma+n+\mu+\delta$

Then the jacobian matrix of $\mathrm{f}$ and $\mathrm{v}$ at the disease free equilibrium are,

$$
\begin{aligned}
& F=\left[\begin{array}{cc}
a b\left(\epsilon v_{0}+s_{0}\right) & a d\left(\epsilon v_{0}+s_{0}\right) \\
(1-a) b\left(\epsilon v_{0}+s_{0}\right) & (1-a) d\left(\epsilon v_{0}+s_{0}\right)
\end{array}\right] ; \\
& V=\left[\begin{array}{cc}
\gamma+\omega+\varepsilon+\mu & 1(1-q) n) \\
-\omega & v+n+\mu+\delta
\end{array}\right]
\end{aligned}
$$

for any $2 \times 2$ matrix sayG $=\left[\begin{array}{ll}a & b \\ c & d\end{array}\right]$, its inverse is obtained by

$A^{-1}=\frac{q}{a d-b c}\left[\begin{array}{cc}d & -b \\ -c & a\end{array}\right]$,

Similarly,

$$
\begin{aligned}
& V^{-1}=\frac{1}{(\gamma+\omega+\varepsilon+\mu)(v+n+\mu+\delta)-\omega n(1-q)} \\
& \times\left[\begin{array}{cc}
\gamma+\omega+\varepsilon+\mu & 1(1-q) n) \\
-\omega & v+n+\mu+\delta
\end{array}\right]
\end{aligned}
$$

then

$$
\begin{aligned}
& F V^{-1}=\frac{1}{(\gamma+\omega+\varepsilon+\mu)(v+n+\mu+\delta)-\omega n(1-q)} \times \\
& {\left[\begin{array}{cc}
a b\left(\epsilon v_{0}+s_{0}\right) v+n+\mu+\delta+a d \omega\left(\epsilon v_{0}+s_{0}\right) & a b\left(\epsilon v_{0}+s_{0}\right)(1-q) n+a d\left(\epsilon v_{0}+s_{0}\right)(\gamma+\omega+\varepsilon+\mu) \\
(1-a) b\left(\epsilon v_{0}+s_{0}\right)\left((v+n+\mu+\delta)+a d\left(\epsilon v_{0}+s_{0}\right) \omega\right. & (1-a) d\left(\epsilon v_{0}+s_{0}\right)(1-q) n+(1-a) d\left(\epsilon v_{0}+s_{0}\right)(\gamma+\omega+\varepsilon+\mu)
\end{array}\right]}
\end{aligned}
$$

Now, to obtain dominant eigenvalue of $\mathrm{FV}^{-1}$

Let

$$
\begin{aligned}
& a_{1}=\frac{(v+n+\mu+\delta)}{(\gamma+\omega+\varepsilon+\mu)(v+n+\mu+\delta)-\omega n(1-q)}\left(\epsilon \mathcal{V}_{0}+s_{0}\right), \\
& a_{2}=\frac{\omega}{(\gamma+\omega+\varepsilon+\mu)(v+n+\mu+\delta)-\omega n(1-q)}\left(\epsilon \mathcal{V}_{0}+s_{0}\right), \\
& a_{3}=\frac{(1-q) n}{(\gamma+\omega+\varepsilon+\mu)(v+n+\mu+\delta)-\omega n(1-q)}\left(\epsilon \mathcal{V}_{0}+s_{0}\right), \\
& a_{4}=\frac{(\gamma+\omega+\varepsilon+\mu)}{(\gamma+\omega+\varepsilon+\mu)(v+n+\mu+\delta)-\omega n(1-q)}\left(\epsilon v_{0}+s_{0}\right), \\
& \mathrm{FV}^{-1} \text { becomes, } \\
& \mathrm{FV}^{-1}=\left[\begin{array}{cc}
a b a_{1}+a b a_{2} & a b a_{3}+a b a_{4} \\
(1-a) b a_{1}+(1-a) d a_{2} & (1-a) b a_{3}+(1-a) d a_{4}
\end{array}\right]
\end{aligned}
$$

The eigenvalue of $\mathrm{FV}^{-1}$ is obtained as follows:

$$
\begin{aligned}
& {\left[\begin{array}{cc}
b a a_{1}+d a a_{2}-\lambda & b a a_{3}+d a a_{4} \\
b(1-a) a_{1}+d(1-a) a_{2} & \left(b(1-a) a_{3}+d(1-a) a_{4}-\lambda\right.
\end{array}\right]} \\
& \left.\rightarrow\left(\left(b a a_{1}+d a a_{2}\right)-\lambda\right)+\left((1-a) b a_{3}+(1-a) d a_{4}\right)-\lambda\right)-\left((1-a) b a_{1}+(1-a) d a_{2}\left(b a a_{3}+d a a_{4}\right)=\right. \\
& 0 \\
& \rightarrow \lambda^{2}-\left(a\left(b a_{1}+d a_{2}\right)\right)+\left((1-a)\left(b a_{3}+d a_{4}\right)\right) \lambda-a\left(b a_{1}+d a_{2}\right)(1-a)\left(b a_{3}+d a_{4}\right)-a(1 \\
& -a)\left(b a_{1}+d a_{2}\right)\left(b a_{3}+d a_{4}\right)=0 \\
& \rightarrow \lambda^{2}-\left(a\left(b a_{1}+d a_{2}\right)\right)+\left((1-a)\left(b a_{3}+d a_{4}\right)\right) \\
& -\left(b a_{1}+d a_{2}\right)\left(b a_{3}+d a_{4}\right)(a((1-a)-a(1-a))=0
\end{aligned}
$$


$\rightarrow \lambda^{2}-\left(a\left(b a_{1}+d a_{2}\right)\right)+\left((1-a)\left(b a_{3}+d a_{4}\right) \lambda\right)=0$

$\lambda\left(\lambda-\left(a\left(b a_{1}+d a_{2}\right)(1-a)\left(b a_{3}+a_{4}\right)=0\right.\right.$

The eigenvalue are,

$\lambda_{1}=0$

$\lambda_{2}=\mathrm{a}\left(\mathrm{ba}_{1}+\mathrm{da}_{2}\right)+(1-\mathrm{a})\left(\mathrm{ba}_{3}+\mathrm{da}_{4}\right)=0$

Among $\lambda_{1}$ and $\lambda_{2}$ the dominant eigenvalue is $\lambda_{2}$

The Reff $=a\left(b_{1}+d_{2}\right)+(1-a)\left(b_{3}+d_{4}\right)$

By substituting the values of $\mathrm{a}_{1}, \mathrm{a}_{2}, \mathrm{a}_{3}, \mathrm{a}_{4}$, are effective reproduction number become

$\operatorname{Reff}=\left[\frac{a(b(v+n+\mu+\delta+\omega)}{(\gamma+\omega+\varepsilon+\mu)((v+n+\mu+\delta+\omega)-\omega n(1-q)}+\frac{(1-b)(b(1-q) n+d(\gamma+\omega+\varepsilon+\mu)}{(\gamma+\omega+\varepsilon+\mu)(v+n+\mu+\delta)-\omega n(1-q)}\right]\left(\epsilon v_{0}-s_{0}\right)$

Put $\mathrm{b}=\mathrm{k} \tau P$ and $\mathrm{d}=\mathrm{k} \tau$ and $\mathrm{X}=\mathrm{k} \tau$,

$\operatorname{Reff}=\mathrm{k} \tau\left[\frac{a(p(b(v+n+\mu+\delta+\omega)}{(\gamma+\omega+\varepsilon+\mu)(v+n+\mu+\delta+\omega)-w n(1-q)}+\frac{(1-b)(p(1-q) n+d(\gamma+\omega+\varepsilon+\mu)}{(\gamma+\omega+\varepsilon+\mu)(v+n+\mu+\delta)-\omega \mathrm{n}(1-\mathrm{q})}\right]\left(\frac{\pi}{\mu} A-\frac{\pi}{\mu} B\right)$

\subsection{Analysis of Reff}

The Reff measure the average number of new infectious generated by a typically infectious individual in a community when some strategies are in place, like vaccination or treatment. The calculated Reff is,

$\operatorname{Reff}=\mathrm{k} \tau\left[\frac{a(p(v+n+\mu+\delta+\omega)}{(\gamma+\omega+\varepsilon+\mu)(v+n+\mu+\delta+\omega)-\omega n(1-q)}+\frac{(1-a)(p(1-q) n+(\gamma+\omega+\varepsilon+\mu)}{(\gamma+\omega+\varepsilon+\mu)(v+n+\mu+\delta)-\omega \mathrm{n}(1-\mathrm{q})}\right] \epsilon \frac{\pi}{\mu} B$

Thus,

$\operatorname{Reff}=R_{S}^{*} A+R_{S}^{*} B$

$R_{S}^{*} A=\epsilon \lambda \mathrm{k} \tau\left[\frac{a(p(v+n+\mu+\delta)+\omega}{(\gamma+\omega+\varepsilon+\mu)(v+n+\mu+\delta)-\omega n(1-q)}+\frac{(1-a)(p(1-q) n+(\gamma+\omega+\varepsilon+\mu)}{(\gamma+\omega+\varepsilon+\mu)(v+n+\mu+\delta)-\omega \mathrm{n}(1-\mathrm{q})}\right] \frac{\pi}{\mu}$

$R_{v}^{*}=\epsilon \mathrm{k} \tau\left[\frac{a(p(v+n+\mu+\delta)+\omega}{(\gamma+w+\omega+\mu)(v+n+\mu+\delta)-\omega n(1-q)}+\frac{(1-a)(p(1-q) n+(\gamma+\omega+\varepsilon+\mu)}{(\gamma+\omega+\varepsilon+\mu)(v+n+\mu+\delta)-\omega \mathrm{n}(1-\mathrm{q})}\right] \frac{\pi}{\mu}$

For the overall disease transmission there is a combination from susceptible population $\left(R_{S}^{*}\right)$ and vaccinated population $\left(R_{v}^{*}\right)$

\section{Definition 3.1}

1. The threshold parameters $R_{S}^{*}$, is the reproduction number when all individuals are vaccinated.

2. The threshold parameter $R_{v}^{*}$ is the reproduction number when all individuals are vaccinated

Let us suppose that initially the entire population is susceptible. This means that $\epsilon=0$, $\mathrm{p}=0, \alpha=0, \pi=0$, then Reff becomes,

$$
\begin{aligned}
& R_{0}=\left[( 1 - a ) \left(\frac{\gamma+\omega+\varepsilon+\mu}{(\gamma+\omega+\varepsilon+\mu)(v+n+\mu+\delta)-\omega n(1-q)}\right.\right. \\
& \left.+(1-q) b \frac{n}{(\gamma+\omega+\varepsilon+\mu)(v+n+\mu+\delta)-\omega n(1-q)}\right) \\
& +a\left(b \frac{(v+n+\mu+\delta)}{(\gamma+\omega+\varepsilon+\mu)(v+n+\mu+\delta)-\omega n(1-q)}\right. \\
& \left.\left.+d \frac{\omega}{(\gamma+\omega+\varepsilon+\mu)(v+n+\mu+\delta)-\omega n(1-q)}\right)\right] \frac{\pi}{\mu}
\end{aligned}
$$

which is the basic reproductive number, means the average number of secondary infectious caused by a single infective in totally susceptible population. 


\subsection{Local stability of disease free equilibrium}

\section{Theorem 3.4}

The disease free equilibrium point if locally asymptomatically stake of $\operatorname{Reff}<1$ and unstable of Reff $>1$.

\section{Proof :}

To prove DFE, Jacobian matrix of the system (1) and (5) at $E_{0}$ are:

$\mathrm{J}\left(\mathrm{V}_{0}, \mathrm{~S}_{0}, 0,0\right)=$
$\left[\begin{array}{cc}-(\alpha+\mu) & \beta \\ \alpha & -(\beta+\mu) \\ 0 & 0 \\ 0 & 0 \\ 0 & 0\end{array}\right.$

So obtain eigenvalues of (11)

Eq. (12) is expanded and simplified

$\lambda^{2}+(2 \mu+\alpha+\beta) \lambda+\mu(\mu+\alpha+\beta)=0$

then by Routh-Hurwitz criteria eq. (13) have strictly negative root.

The determinant of eq. (13) can be obtained as 
$-(\varnothing+\sigma+\mu)$

$\lambda\left[\begin{array}{cc}\epsilon a b v_{0}+a b s_{0}-(\gamma+\omega+\varepsilon+\mu)-\lambda & \epsilon a d v_{0}+a d s_{0}+(1-q) n \\ (1-a) \epsilon b v_{0}+(1-a) b s_{0}+\omega & (1-a) \epsilon d v_{0}+(1-a) d s_{0}-(v+n+\mu+\delta)-\lambda\end{array}\right]$

Then $\lambda^{2}=-(\varnothing+\sigma+\mu)<0$

and $\quad\left(\epsilon a b v_{0}+a b s_{0}-(\gamma+\omega+\varepsilon+\mu)-\lambda\right)(1-a) \epsilon d v_{0}+(1-a) d s_{0}-(v+n+\mu+\delta)-$ $\left.\lambda(1-a) \epsilon b v_{0}+(1-a) b s_{0}+\omega\right) \in a d v_{0}+a d s_{0}+(1-q) n=0$

$\left(a b\left(\epsilon v_{0}+s_{0}\right)-(\gamma+\omega+\varepsilon+\mu)(1-a) d\left(\epsilon v_{0}+d s_{0}\right)-(v+n+\mu+\delta) \lambda\left((1-a) b\left(\epsilon v_{0}+\right.\right.\right.$

$\left.\left.s_{0}\right)+\omega\right)\left(a d\left(\in v_{0}+s_{0}\right)(1-q) n=0\right.$

Rearranging eq. (14) it becomes,

$\lambda^{2}+\mathrm{a}_{1} \lambda+\mathrm{a}_{2}=0$

where

$\mathrm{a}_{1}=(\gamma+\omega+\varepsilon+\mu)+(v+n+\mu+\delta)-(\mathrm{ab}+(1-\mathrm{a}) \mathrm{d})\left(\in v_{0}+s_{0}\right)$

$\mathrm{a}_{2}=-\left(a(b(v+n+\mu+\delta)+\mathrm{d} \omega)+(1-\mathrm{a})(\mathrm{d}(b(\gamma+\omega+\varepsilon+\mu)+\mathrm{b}(1-\mathrm{q}) \mathrm{n}))\left(\in v_{0}+s_{0}\right)-\right.$ $((1-\mathrm{q}) \omega \mathrm{n}-((\gamma+\omega+\varepsilon+\mu)(v+n+\mu+\delta))$

By Routh-Hurwitz Criteria $\mathrm{a}_{1}>0$

means that

$$
\begin{gathered}
(a(b(v+n+\mu+\delta)+\mathrm{d} \omega)+(1-\mathrm{a})(\mathrm{d}(\gamma+\omega+\varepsilon+\mu)+\mathrm{b}(1-\mathrm{q}) \mathrm{n}))\left(\in v_{0}+s_{0}\right) \\
<(\gamma+\omega+\varepsilon+\mu)(v+n+\mu+\delta))-(1-\mathrm{q}) \\
(a(b(v+n+\mu+\delta)+\mathrm{d} \omega)+(1-\mathrm{a})(\mathrm{d}(\gamma+\omega+\varepsilon+\mu)+\mathrm{b}(1-\mathrm{q}) \mathrm{n}))\left(\in v_{0}+s_{0}\right) \\
<(\gamma+\omega+\varepsilon+\mu)(v+n+\mu+\delta))-(1-\mathrm{q}) \\
\frac{(a(b(v+n+\mu+\delta)+\mathrm{d} \omega)+(1-\mathrm{a})(\mathrm{d}(\gamma+\omega+\varepsilon+\mu)+\mathrm{b}(1-\mathrm{q}) \mathrm{n}))\left(\in v_{0}+s_{0}\right)}{(\gamma+\omega+\varepsilon+\mu)(v+n+\mu+\delta))-(1-\mathrm{q}) \omega \mathrm{n}}<1
\end{gathered}
$$

i.e., $\operatorname{Reff}<1$

Thus the disease free equilibrium is locally asymptomatically stable if $\operatorname{Reff}<1$.

\subsection{The endemic equilibrium (EF)}

The endemic equilibrium is defined as a steady state solution of the model and it is denoted by $E^{*}$. It means that the disease persists. It is obtained by equating the system (1)(5) to zero.

Taking equation third and fourth of the model, we get,

$C^{*}=\frac{(1-a)(1-q) n+a(v+n+\mu+\delta)}{(1-a)((\gamma+\omega+\varepsilon+\mu))+a \omega} I^{*}$

From the first equation of the model,

$\mathrm{V}^{*}=\frac{P \pi+\beta s^{*}}{\mu+\epsilon \lambda+\alpha}$

by combining (15) and the third and fourth equation of the model we obtain,

$S^{*}=\frac{((\gamma+\omega+\varepsilon+\mu))((1-a)(1-q) n+a v+n+\mu+\delta-a(1-q) n((1-a)(\gamma+\omega+\varepsilon+\mu)+a \omega)(\mu+\epsilon \lambda+\alpha)}{(((1-a)(\gamma+\omega+\varepsilon+\mu)+a \omega)(\mu+\emptyset+\sigma)) a \lambda(\mu+\epsilon \lambda+\alpha+\in \beta)} I^{*}$

$R^{*}=\frac{\varepsilon((1-a)(1-q) n+a(v+n+\mu+\delta)+((1-a)(\gamma+\omega+\varepsilon+\mu)+a \omega) q n}{(((1-a)(\gamma+\omega+\varepsilon+\mu)+a \omega)(\mu+\emptyset+\sigma)) a} I^{*}$

From (15), (16), (17) and second equation of the model we get,

$R^{*}=\frac{A_{2} \lambda^{* *}\left(\lambda^{* * 2} \epsilon^{2} D_{2}+\mu \in D_{2}+\lambda^{* *} \in D_{2}+\lambda^{* *} \in D_{2} D_{6}+\lambda^{* *} \in D_{4}+\mu D_{2} D_{6}+\emptyset D_{2} D_{6}-\lambda^{* *} D_{2}+D_{1} D_{7}+D_{4} D_{7}\right)}{A_{1}\left(\lambda^{* *} \in D_{5}\right) D_{7}-\delta \lambda^{* * 3} A_{5} A_{2} \epsilon^{2}+\delta \lambda^{* * 2} D_{5} A_{5} \in+\delta \lambda^{* *} D_{6} D_{5} A_{5} A_{2}+A_{1}\left(\lambda^{* *} \in+D_{5}\right)}$

Where

$\mathrm{A}_{1}=(\gamma+\omega+\varepsilon+\mu)((v+n+\mu+\delta)-\mathrm{n}(1-\mathrm{q})$,

$\mathrm{A}_{2}=\mathrm{a} \omega+(1-a)(\gamma+\omega+\varepsilon+\mu)$ 


$$
\begin{aligned}
& \mathrm{A}_{3}=\mathrm{a}(\mu+\emptyset+\sigma)^{2}(\omega+(1-a)(\gamma+\omega+\varepsilon+\mu)) \\
& \mathrm{A}_{4}=\mathrm{a} \omega+(1-a)(\mu+\emptyset+\sigma), \\
& \mathrm{A}_{5}=((1-\mathrm{a}) \mathrm{n}(1-\mathrm{q})+\mathrm{a}(\gamma+\omega+\varepsilon+\mu)+(\omega a+(1-a) \mathrm{a}(\gamma+\omega+\varepsilon+\mu) \mathrm{qn} \\
& \mathrm{D}_{1}=\in P \pi \\
& \mathrm{D}_{2}=A_{3}(1-P) \pi \\
& \mathrm{D}_{3}=A_{3} \beta P \pi \\
& \mathrm{D}_{4}=\mu+\alpha \\
& \mathrm{D}_{5}=\in \beta+\mu+\alpha \\
& \mathrm{D}_{7}=A_{4} \alpha \beta-\mu-\beta
\end{aligned}
$$

Hence $E^{*}=\left(V^{*}, S^{*}, C^{*}, I^{*}, R^{*},\right)$ is the endemic equilibrium of the model (1)-(5).

\section{Lemma 3.1}

For Reff $>1$ a unique endemic equilibrium point $E^{*}$ exist and no endemic equilibrium otherwise.

\subsection{Sensitivity analysis of the model parameters}

Sensitivity analysis identifies the parameter that has a high impact on the reproduction number (Reff). The normalized forward sensitivity index of a variable to a parameter is a ratio of the relative change in the variable to the relative change in the parameter [13]. If a variable is a differentiable function of the parameter the sensitivity index may be alternately defined using partial derivatives. The sensitivity index can be calculated for all the parameters by using the formula:

$\Delta_{p}^{u}=\frac{\delta \mathrm{u}}{\delta \mathrm{p}} \frac{\mathrm{u}}{\mathrm{u}}$ eg. $\Delta_{K}^{\text {Reff }}=\frac{\delta \text { Reff }}{\delta \mathrm{k}} \frac{\mathrm{k}}{\delta \text { Reff }}=1$ or $\Delta_{p}^{\text {Reff }}=\frac{\delta \text { Reff }}{\delta \mathrm{p}} \frac{\mathrm{p}}{\delta \text { Reff }}$

The values of parameters involved in Reff are as follows:

Table 2. Desciption of parametric values.

\begin{tabular}{clcl}
\hline Parameters & Interpretation & Parameters & Interpretation \\
\hline $\mathrm{K}$ & 6 & $v$ & 0.2 \\
$\epsilon$ & 0.4 & $\mathrm{a}$ & 0.338 \\
$p$ & 06 & $\mu$ & 0.002 \\
$\alpha$ & 0.001 & $\delta$ & 0.33 \\
$\beta$ & 0.9 & $\varnothing$ & 0.01 \\
$\omega$ & 1 & $\gamma$ & 0.1 \\
$\mathrm{q}$ & 0.5 & $\sigma$ & 0.05 \\
$n$ & 0.0238 & $\tau$ & 0.89 \\
$\varepsilon$ & 0.0115 & $v$ & 0.2 \\
\hline
\end{tabular}

The values are taken from literature [14,15] and some are assumed and its calculated sensitivity indices are as follows. 
Table 3. Sensitivity indices of parameters.

\begin{tabular}{clcl}
\hline Parameters & Interpretation & Parameters & Interpretation \\
\hline $\mathrm{K}$ & $+\mathrm{ve}$ & $\varepsilon$ & -ve \\
$\epsilon$ & $+\mathrm{ve}$ & $v$ & -ve \\
$p$ & $-\mathrm{ve}$ & $\mathrm{a}$ & $-\mathrm{ve}$ \\
$\alpha$ & $+\mathrm{ve}$ & $\mu$ & $-\mathrm{ve}$ \\
$\beta$ & $-\mathrm{ve}$ & $\tau$ & $+\mathrm{ve}$ \\
$\omega$ & $+\mathrm{ve}$ & $\delta$ & $-\mathrm{ve}$ \\
$\mathrm{q}$ & $-\mathrm{ve}$ & $\gamma$ & $-\mathrm{ve}$ \\
$n$ & $-\mathrm{ve}$ & & \\
\hline
\end{tabular}

\subsubsection{Representation of sensitivity indices}

From the values of the parameter which are given in Table 2, we have calculated the sensitivity indices in the Table 3 from which we conclude that $K, \in, \alpha, \omega$ and $\tau$ are increased keeping other parameters constant.They increased the value of Reff, thus they increased the endemicity of the disease as they have positive indices. Whereas the parameters p, q, $n, \varepsilon, v, a, \mu, \delta, \emptyset, \gamma, \sigma, \beta$ decreased the value of Reff when they are increased while keeping the other parameters constant, which implies that they decreased the endemicity of the disease as they have negative indices.

\section{Discussion}

The parameters which play the significant role in making the reproduction number less than unity which is calculated that could lead to elimination of pneumonia. If these parameters can be controlled by adopting some of the precautionary measures like school deworming programs and safe use of probiotics under medical supervisions at susceptible and carrier classes can help strategically in control programs. These methods are cheap, safe and effective at screening level. It could be a preventive measure and could also help in reducing the severity of pneumonia and COVID-19 to some extent. This will help in boosting the natural immunity of the body. Some of the measures are suggested based on our model where further research and investigation can be done in this direction as an alternate method in the present time of crisis.

\section{Conclusion}

The model of pneumonia was formulated and analyzed. The invariant set in which the solutions of the model is biologically meaningful was derived. Also boundedness of solution was proved. Analysis of the model showed that there exist two possible solutions, namely the DFE and endemic equilibrium point. Further analyses showed that the disease free point is locally stable if $\operatorname{Reff}<1$ or otherwise unstable if Reff $>1$ that leads endemic equilibrium. The sensitivity indices showed the parameter which leads to increase in Reff are $K, \in, \alpha, \omega$ and $\tau$. If these factors are reduced i.e., contact rate $K$, rate at which vaccinated getting transferred to carrier and infected classes i.e., $\epsilon$, waning rate of vaccine 
$\alpha$, rate at which carrier transfers to infected class $\omega$ and $\tau$ i.e., the probability that a contact is effective to cause infection, that could lead to elimination of pneumonia.

\section{References}

1. J. Liu and T. Zhang, Mathematical Comput. Model. 54, 836 (2011). https://doi.org/10.1016/j.mcm.2011.03.033

2. A. Cheepsattayakorn, R. Cheepsattayakorn, Biomed. Res. Int. 2014, ID 874021 (2014). http://dx.doi.org/10.1155/2014/874021

3. B. Nazario, Coronavirus and Pneumonia, https://www.webmd.com (date of access, 22 May, 2020).

4. L. Caly, J. D. Druce, M. G. Catton, D. A. Jans, and K. M. Wagstaff, Antiviral Res. 178, ID 104787 (2020). https://doi.org/10.1016/j.antiviral.2020.104787

5. D. J. Cook, J. Johnstone, J. C. Marshall, F. Lauzier, L. Thabane, S. Mehta, P. M. Dodek, L. Mclntyre, J. Pagliarello, W. Henderson, R. W. Taylor, R. Cartin-Ceba, E. Golan, M. Herridge, G. Wood, D. Ovakim, T. Karachi, M. G. Surette, D. M. E. Bowdish, D. Lamarche, C. P. Verschoor, E. H. Duan, D. H. Ansdell, Y. Arabi, M. Meade, and the Canadian Critical Care Trials Group, BioMed. Central, (2016). https://doi.10.1186/s13063-016-1495-x (Date of access, 25 May, 2020).

6. UNICEF and WHO, Pneumonia the Forgotten Killer of Children (2006).

7. V. Singh and S. Aneja, Pediatric Respiratory Rev. 12, 52 (2011). https://doi.org/10.1016/j.prrv.2010.09.011

8. S. S. Huang, J. A. Finkelstein, and M. Lipsitch, Clin. Inf. Disease 40, 1215 (2005). https://doi.org/10.1086/428580

9. M. Lipsitch, C. Coligri, T. Coher, W.P. Hanage, and C. Fraser, Epidemics 1, 2 (2009). https://doi.org/10.1016/j.epidem.2008.07.001

10. O. J. Otieno, M. Joseph, and O. John, 'Mathematical Model for Pneumonia Dynamics among Children - Southern Africa Mathematical Sciences Association Conference (Samsa 2012).

11. P. V. d. Driessche and J. Watmough, Mathematical Biosci. 180, 29 (2002). https://doi.org/10.1016/S0025-5564(02)00108-6

12. O. Diekmann and J. A. P. Heesterbuk, Int. J. Epidemiology 30, 186 (2001). https://doi.org/10.1093/ije/30.1.186

13. N. Chitnis, J. M. Hyman, and J. M. Cushing, Bull. Mathematical Biol. 70, 1272 (2008). https://doi.10.1007/s11538-008-9299-0

14. C. G. Ngari, D. M. Malowza, and B. G. Muthuri, J. Life Sci. Res. 1, 31 (2014). http://asianonlinejournals.com/index.php/lifsc/index

15. M. Kizito and J. Tumwiine, J. Appl. Math. 2018, ID 2539465 (2018). http://doi.org/10.1155/2018/2539465 